\title{
Qualidade de vida após artroplastia total do joelho: revisão sistemática
}

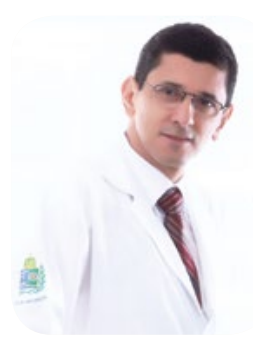

\author{
Robson Rocha da Silva ${ }^{1}$, Ayrton André Melo Santos ${ }^{1}$, José de Sampaio \\ Carvalho Júnior ${ }^{1}$, Marcos Almeida Matos ${ }^{1}$.
}

\begin{abstract}
Artigo original: Robson Rocha da Silva, Ayrton André Melo Santos, José de Sampaio Carvalho Júnior, Marcos Almeida Matos. Publicado na Revista Brasileira de Ortopedia; 2014;49(5):520-527.
\end{abstract}

\section{INTRODUÇÃO}

A doença articular degenerativa é, no geral, a principal causa de deficiência física entre idosos. A dor e limitação funcional provocada por essa condição, especialmente nos membros inferiores, apresenta forte correlação com a redução na qualidade de vida (QV) dessas pessoas. No caso da artropatia degenerativa do joelho, a Artroplastia Total do Joelho (ATJ) é a opção terapêutica de escolha para os casos mais graves.

Os métodos atuais de avaliação dos resultados da ATJ são baseados principalmente em sinais e sintomas clínicos, na avaliação do exame físico e na avaliação radiográfica. Este tipo de avaliação não contempla todos os aspectos do tratamento.

Recentes estudos mostram que fatores subjetivos afetam a forma como os indivíduos internalizam seus sintomas e sua capacidade funcional e, consequentemente, uma avaliação médica objetiva, fundamentada em exame clínico e radiográfico, pode ser menos importante do que a ideia pessoal e individual de que $o$ tratamento foi bem-sucedido.

Diversos estudos revelam melhora na QV dos pacientes submetidos à ATJ. Porém, a utilização de múltiplos instrumentos, intervalos e algoritmos de pontuações diferentes e apresentações de resultados de forma não padronizada também têm levado a desafios no entendimento da literatura sobre este tema. Este estudo tem como objetivo revisar a literatura sobre QV em pacientes submetidos à ATJ.

\section{MATERIAL E MÉTODOS}

A fim de levantar a literatura a respeito do assunto, foi realizada uma pesquisa nos bancos de dados MEDLINE, EMBASE, LILACS e ScIELO, utilizando-se os termos: TKA (total kneearthroplasty);TKR (total kneereplacement); qualityoflife; e outcomes. Os estudos foram classificados em grau de evidência baseado no CEBM8.
Os aspectos avaliados nos estudos selecionados foram de cunho metodológico, tais como autoria, desenho do estudo, ano de realização, população alvo e amostra, instrumento de coleta de dados utilizado e desfechos clínicos principais. Também foi realizada uma avaliação crítica dos instrumentos utilizados, dos fatores que poderiam atuar como variáveis de confundimento e a provável relação entre qualidade de vida e expectativas dos pacientes.

\section{RESULTADOS}

A busca gerou um total de 31 artigos selecionados, sendo 28 observacionais e 03 estudos de revisão (02 revisões sistemáticas e uma revisão narrativa).

Tabela 1 - Níveis de evidência e desenho dos estudos selecionados.

\begin{tabular}{|ccccc}
\hline \hline ID & Autor & Ano & $\begin{array}{c}\text { Nível de } \\
\text { Evidência }\end{array}$ & Desenho \\
\hline 1 & Narayanasamy et al. & 2011 & Nível 2B & Coorte prospectivo \\
\hline 2 & Santic et al. & 2012 & Nível 2B & Coorte prospectivo \\
\hline 3 & Papakostidou et al. & 2012 & Nível 2B & Coorte prospectivo \\
\hline 4 & Scott et al. & 2012 & Nível 2B & Coorte prospectivo \\
\hline 5 & GrosseFrie et al. & 2012 & Nível 2B & Coorte prospectivo \\
\hline 6 & Baker et al. & 2012 & Nível 2B & Coorte prospectivo \\
\hline 7 & Schwartz et al. & 2012 & Nível 2B & Coorte prospectivo \\
\hline 8 & Zhang et al. & 2012 & Nível 2B & Coorte prospectivo \\
\hline 10 & Desmeules et al. & 2010 & Nível 2B & Coorte prospectivo \\
\hline 11 & Bugala-Szpak et al. & 2010 & Nível 2B & Coorte prospectivo \\
\hline 12 & Kauppila et al. & 2010 & Nível 2B & Coorte prospectivo \\
\hline 13 & Gawel et al. & 2010 & Nível 2B & Coorte prospectivo \\
\hline 14 & Brandes et al & 2010 & Nível 2B & Coorte prospectivo \\
\hline 15 & Desmeules et al. & 2012 & Nível 2B & Coorte prospectivo \\
\hline 17 & Baumann et al. & 2011 & Nível 2B & Coorte prospectivo \\
\hline 16 & Ackerman et al. & 2011 & Nível 2B & Coorte prospectivo \\
\hline & & & & \\
\hline
\end{tabular}




\begin{tabular}{lcccc}
\hline 17 & Gonzalez et al. & 2010 & Nível 2B & Coorte prospectivo \\
\hline 18 & Kilic et al. & 2009 & Nível 2B & Coorte prospectivo \\
\hline 19 & Nunez et al. & 2009 & Nível 2B & Coorte prospectivo \\
\hline 20 & Loughead et al. & 2008 & Nível 2B & Coorte prospectivo \\
\hline 21 & McHugh et al. & 2008 & Nível 2B & Coorte prospectivo \\
\hline 22 & Fitzgerald et al. & 2004 & Nível 2B & Coorte prospectivo \\
\hline 23 & Greidanus & 2011 & Nível 2B & Coorte retrospectivo \\
\hline 24 & Rissanen et al. & 1996 & Nível 2B & Coorte prospectivo \\
\hline 25 & Nunez M et al. & 2007 & Nível 2B & Coorte prospectivo \\
\hline 26 & Bruyere O et al. & 2012 & Nível 2B & Coorte prospectivo \\
\hline 27 & Lingard et al. & 2004 & Nível 2B & Coorte prospectivo \\
\hline 28 & Scott et al. & 2010 & Nível 2B & Coorte prospectivo \\
\hline 29 & Jones et al. & 2012 & Nível 2-A & Revisão Sistemática \\
\hline 30 & Vissers et al. & 2012 & Nível 2-A & Revisão Sistemática \\
\hline 31 & Ethgen O. et al. & 2004 & Nível 2-A & Revisão Sistemática \\
\hline \hline
\end{tabular}

\section{DISCUSSÃO \\ Questionários para avaliação da qualidade de vida}

Todos os estudos avaliados referiram melhora na QV dos pacientes submetidos à artroplastia total do joelho. Contudo, diversos aspectos foram avaliados e vários métodos e protocolos diferentes foram utilizados.

Dos vários aspectos avaliados que foram associados ao conceito de qualidade de vida, um dos mais frequentes foi a função. Gawel et. al., em estudo observacional, apresentaram que significativa melhora na função do joelho foi observada nos pacientes quando caminhando, subindo escadas e na estabilidade. Estes achados positivos foram observados já na quarta semana de avaliação. Fitzgerald et. al., entretanto, constataram que um mês após a cirurgia foi observado importante decréscimo na função física, fato que aumentou a dependência do apoio familiar.

Brandes et al. constataram que a ATJ oferece profunda melhora e excelentes resultados clínicos para a maioria dos pacientes. O nível de atividade após o tratamento parece ser mais influenciado pelo hábito de praticar atividade física antes da cirurgia do que pelo próprio tratamento.

Com respeito à dor, foi observada melhora em diversos estudos. Os efeitos positivos da cirurgia puderam ser observados logo em uma, quatro e seis semanas no período pós-operatório, e perduraram por um período de até sete anos após a cirurgia. A melhora na dor apresentou íntima correlação com a conquista de melhores escores na QV, sendo que a permanência da dor nas avaliações pós-operatórias reduziu a possibilidade de alcançar bons resultados.

\section{Aspectos sóciodemográficos que influenciaram na qualidade de vida}

Com respeito ao gênero, segundo Papakostidou et al., pacientes do sexo feminino apresentaram escores inferiores tanto na avaliação pré-operatória quanto em seis semanas após a cirurgia. Outro estudo constatou que tanto a idade avançada como a doença pulmonar reduziram a possibilidade de alcançar QV satisfatória. A escolaridade não interferiu na QV dos pacientes submetidos à ATJ.

Obesidade e complicações pós-operatórias foram associadas com os piores escores em todas as dimensões do WOMAC. Escores pós-operatórios baixos do WOMAC ocorreram na presença de obesidade severa, havendo prejuízo significativo com respeito à dor, rigidez e escores funcionais.

Tempo de espera para a cirurgia e sua correlação com a QV foi estudada em alguns dos artigos selecionados. Desmeules et. al. observaram que a longa espera pela cirurgia tem impacto significativamente negativo na dor, função e QV. Após seis meses de espera, significativa diferença foi observada entre os grupos com relação à QV, pacientes com espera maior que nove meses apresentaram os piores escores.

Relação entre nível de expectativa, satisfação pós-operatória e qualidade de vida.

A realização das expectativas pré-operatória foi altamente correlacionada com o grau de satisfação. Pacientes que referiram ter suas expectativas alcançadas numa avaliação após 12 meses da cirurgia tiveram também um maior e significativo ganho na $\mathrm{QV}$. Aqueles que tiveram suas expectativas atingidas tiveram um grande ganho na $Q V$.

Scott et. al. constataram que as expectativas dos pacientes tiveram alta correlação com satisfação após um ano de cirurgia. Referiram que o manejo das expectativas e da saúde mental dos pacientes pode reduzir a insatisfação. Pacientes satisfeitos com a informação médica sobre a cirurgia tiveram altos escores pós-operatórios de QV.

\section{Perspectivas do estudo}

Nosso estudo revelou a necessidade de padronização das escalas de qualidade de vida. A padronização pode melhorar a utilização das informações provenientes deste tipo de pesquisa. A avaliação do paciente submetido à ATJ não pode se limitar à sua condição 
de saúde, mas deve incluir seus sentimentos, expectativas relacionadas à sua função para atividades da vida diária.

\section{CONCLUSÃO}

A artroplastia total de joelho é um procedimento capaz de melhorar globalmente a qualidade de vida dos pacientes. A dor e a função estão entre os mais importantes preditores de melhoria da QV. Outros fatores que estiveram associados positivamente com QV após ATJ foram a melhora do equilíbrio dinâmico e da claudicação, melhora na qualidade do sono, prática de atividade física anterior ao procedimento, suporte sociofamiliar adequado e preenchimento das expectativas do paciente em relação aos resultados da cirurgia. Os fatores associados negativamente foram obesidade, idade avançada, comorbidades, persistência de dor após o procedimento e espera demorada para realização da cirurgia.

1- Serviço de Ortopedia e Traumatologia do HSI.

Endereço para correspondência:

contato@ortopediahsi.com.br 